\title{
A Modified Damage Law with the Fuzzy Neural Network Method for Crack Initiation Life Prediction of Notched Specimen
}

\author{
Jiaying GAO*, Peng LI**, Qiuyang HE*** \\ *School of Aeronautics Science and Engineering, BeiHang University, Beijing 100191, China, \\ E-mail: gaojiaying@buaa.edu.cn \\ **School of Aeronautics Science and Engineering, BeiHang University, Beijing 100191, China, \\ E-mail: lpeng210@sina.com \\ ***Department of Computer Science, The University of York, York, U.K., E-mail: heqiuyang926@163.com \\ cross $^{\text {ref }}$ http://dx.doi.org/10.5755/j01.mech.23.6.14862
}

\section{Introduction}

In practical application, many engineering structures are subject to cyclic load and fatigue failure is a common form of failure [1], which is also a famous technical problem. Most of the time, the maximal value of cyclic load is far lower than the static strength of material. So it is very important to study the fatigue life prediction method.

In the past decades, many researchers have been focused on the fatigue experiment and statistic analysis [2], which is a general method of fatigue life prediction in engineering. However, this method takes too much time and sometimes the result is not satisfactory. So it is necessary to find an easy and reliable method. Fatigue accumulation damage theory $[3,4]$ is considered to be one of the most effective methods for fatigue life prediction. Since 1988, many kinds of fatigue damage models have been established by researchers [5-7] and the damage mechanics based method have been widely used in the engineering application [8-11].

However, it is still difficult to predict the fatigue life of notched specimen because a great number of factors can affect the result. In high cycle fatigue, the prediction accuracy often does not satisfy the design requirements and there are many parameters in the damage evolution law which are difficult to demarcate. Thus there exist differences between the actual life of the material and the theoretical one. The reason is complicated. On one hand, the theoretical model has its own shortage. The estimation of parameters and the expression of empirical formula cannot satisfy all real situations. On the other hand, the characteristics of material and conditions of environment, hardware in experiment caused systematic error. It is not enough to predict the actual life of material simply through the traditional theoretical analysis.

Therefore, the continuum damage mechanics based method with Hence the fuzzy neural network method is the fuzzy neural network method is proposed to solve this problem and to obtain a more reliable life. Fuzzy neural network has demonstrated its superiority in pattern recognition, intelligent control, prediction and other engineering fields [12-14]. It has been proved theoretically that the network consists of 3 layers (an input layer, a hidden layer and an output layer) is able to approximate any nonlinear continuous function with any accuracy. In dealing with complex problems, using NN method has the following advantages: obtaining the implicit expression of non-linear and non-smooth the relationship; good fault tolerance and high forecasting accuracy; Strong ability of association, memory and generalization. These characteristics of the NN method makes it play an important role in the modeling of the complex systems such as life prediction [15]. XL Liao developed a NN model to forecast the tensile fatigue life of carbon material [16]. Cecchi, A combined the ANSYS and a fuzzy NN model to design the mixed flow pump impeller blades [17]. DJ Armaghani built an ICA-ANN model to predict the strength and elasticity modulus of granite [18].

In this paper, according to the damage evolution law of Lemaitre, a modified damage evolution model combined with the fuzzy neural network method to predict the fatigue life of notched specimen is proposed. Then the method to obtain material parameters from the present fatigue experimental data in the modified equation is proposed. At last, the fatigue life prediction for notched plate is conducted and the calculated results with the proposed method are compared with the experimental results. Although the life prediction after being modified may not be accurate in every local region, the combination between the theoretical method and the fuzzy neural network method will obtain a more reliable material life in the whole.

\section{The modified damage evolution law}

\subsection{The modified initial damage threshold}

In high cycle fatigue, as the stress level is not very high, the materials or structure generally does not produce plasticity deformation on the macro scale, which means no damage appears. However, according to the damage accumulation theory of Lemaitre, the micro plasticity deformation will generate as long as the stress exceeds the fatigue limit of material, which will lead to the damage accumulation. Thus the damage accumulation can be measured by the threshold value of the cumulative micro plastic von mises strain. Lemaitre [19] gives the following formula:

$$
p^{\mu}<p_{D}=\varepsilon_{p D}\left(\frac{\eta}{C_{y}}\right)^{m}\left[\frac{\sigma_{u}-\sigma_{f}}{\left(\Delta \sigma_{\Sigma} / 2\right)-\sigma_{f}}\right] .
$$

where $p$ is the accumulated plastic strain; $p_{D}$ is the damage threshold accumulated plastic strain; $\varepsilon$ is the damage 
threshold plastic strains; $\sigma_{u}$ is the ultimate stress; $\sigma_{f}$ is fatigue limit; $\Delta \sigma / 2$ is the stress amplitude and $\mathrm{m}$ is material parameter.

However, there is a problem of Eq. (1) when conducting the fatigue life prediction. In high cycle fatigue, the stress amplitude is close to the fatigue limit, so the term of $\left(\Delta \sigma_{\Sigma} / 2-\sigma_{f}\right)$ will change sharply as the stress amplitude increase or decrease slightly, which will lead to the great change of the predicted fatigue life and it does not agree with the real situation.

In this paper, based on the fatigue experimental data of LC4 plate, the expression of $p$ is modified as follows:

$$
p<p=\varepsilon\left(\frac{\sigma-\alpha \sigma}{\Delta \sigma / 2}\right), 0<\alpha<1, m>0 .
$$

Damage will initiate after a number of cycles $N O$ when the damage threshold $p$ is reached on the micro scale. There is then no micro damage as long as $N<N O$. Considering a periodic loading, we have:

$$
N_{0}=\frac{p_{D}}{2\left|\Delta \varepsilon_{p \Sigma}^{\mu}\right|}=\frac{E p_{D}}{2 \Delta \sigma_{\Sigma}} .
$$

\subsection{Simplified damage evolution equation}

In high cycle fatigue, the stress-strain curve at two sacles (meso scale and micro scale) is shown in Fig. 1 [19].

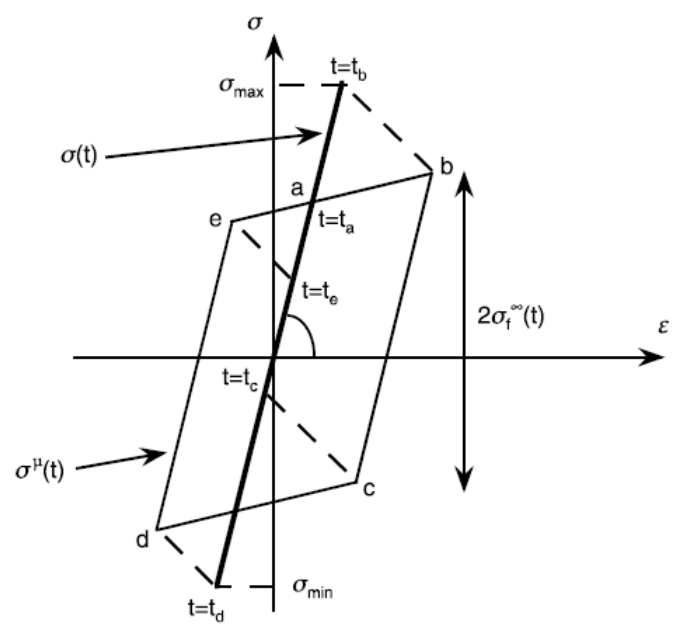

Fig. 1 The stress-strain cycles at micro scale and at meso scale

The thick line represents the stress strain curve at meso scale, which means no macro plasticity produced. The thin line represents the stress-strain curve at micro scale.

In the micro stress-strain cycle, when the damage is accumulated during the elastic-plastic period, Lemaitre considers:

$$
\tilde{\sigma}_{e q}^{\mu}=\frac{\sigma_{e q}^{\mu}}{1-D} \approx \sigma_{f} .
$$

$\sigma_{f}$ represents the fatigue limit. With $\dot{\varepsilon}_{p \Sigma}^{\mu}=\dot{\sigma} / \eta, \eta \approx 3 G(1-\beta)$, the expression of damage evolution law can be simplified as follows:

$$
\begin{aligned}
\dot{D} & =\left(\frac{Y^{\mu}}{S}\right)^{s} \dot{p}^{\mu}=\left(\frac{Y^{\mu}}{S}\right)^{s} \dot{\varepsilon}_{p \Sigma}^{\mu}=\left(\frac{Y^{\mu}}{S}\right)^{s} \frac{\dot{\sigma}_{\Sigma}^{\mu}}{\eta}= \\
& =\left(\frac{\sigma_{f}^{2}}{2 E S}\right)^{s}\left(R_{v}^{\mu}\right)^{s} \frac{\dot{\sigma}_{\Sigma}^{\mu}}{\eta} .
\end{aligned}
$$

In the upper branch of the cycle, the damage increment is:

$$
\delta D_{1}=\left(\frac{\sigma_{f}^{2}}{2 E S}\right)^{s} \frac{1}{\eta} \int_{\sigma_{\Sigma}(e)}^{\sigma_{\Sigma}(b)}\left(R_{v}^{\mu}\right)^{s} d \sigma_{\Sigma} .
$$
crement is:

In the lower branch of the cycle, the damage in-

$$
\delta D_{2}=\left(\frac{\sigma_{f}^{2}}{2 E S}\right)^{s} \frac{1}{\eta} \int_{\sigma_{\Sigma}(d)}^{\sigma_{\Sigma}(c)}\left(R_{v}^{\mu}\right)^{s} d \sigma_{\Sigma} .
$$

Then the damage increment over one cycle is:

$$
\frac{\delta D}{\delta N}=\delta D_{1}+\delta D_{2}=\frac{\sigma_{f}^{2 s}}{(2 E S)^{s} \eta}\left[\begin{array}{l}
\int_{\sigma_{\Sigma}(e)}^{\sigma_{\Sigma}(b)}\left(R_{v}^{\mu}\right)^{s} d \sigma_{\Sigma}+ \\
+\int_{\sigma_{\Sigma}(d)}^{\sigma_{\Sigma}(c)}\left(R_{v}^{\mu}\right)^{s} d \sigma_{\Sigma}
\end{array}\right]
$$
equation:

Eq. (8) can be approximated by the following

$$
\left\{\begin{array}{l}
\frac{\delta D}{\delta N}=\frac{2}{\eta}\left[\frac{(1+v) \sigma_{f}^{2}}{3 E S}\right]^{s} \times \\
\times\left[\sigma_{e q \max }-\sigma_{e q \min }-\sigma_{f}\right], \text { if } \sum_{k k}=0(\text { e.g.shear }) ; \\
\frac{\delta D}{\delta N}=\delta D_{1}+\delta D_{2}=\frac{\sigma_{f}^{2 s}}{(2 E S)^{s} \eta} \times \\
\times\left[\sigma_{e q \max }-\sigma_{e q \min }-\frac{2 \sigma_{f}}{\sum_{k k}}\right]\left[R_{v \min }^{s}+R_{v \max }^{s}\right], \text { if } \sum_{k k} \neq 0 .
\end{array}\right.
$$

Finally, we get:

$$
\left\{\begin{array}{l}
\frac{\delta D}{\delta N}=\frac{4(1+v)}{3 E(1-\beta)}\left[\frac{(1+v) \sigma_{f}^{2}}{3 E S}\right]^{s} \times \\
\times\left[\Delta \sigma_{e q}-\sigma_{f}\right], \text { if } \sum_{k k}=0 ; \\
\frac{\delta D}{\delta N}=\frac{2(1+v) \sigma_{f}^{2 s}}{3 E(1-\beta)(2 E S)^{s}}\left[\Delta \sigma_{e q}-\frac{2 \sigma_{f}}{\sum_{k k}}\right] \times \\
\times\left[R_{v \text { min }}^{s}+R_{v \text { max }}^{s}\right], \text { if } \sum_{k k} \neq 0 .
\end{array}\right.
$$

For the uniaxial fatigue, $\Sigma_{k k}=1$, the damage evolution equation becomes:

$$
\begin{aligned}
& \frac{\delta D}{\delta N}=\frac{2(1+v) \sigma_{f}^{2 s}}{3 E(1-\beta)(2 E S)^{s}} \times \\
& \times\left[\Delta \sigma-2 \sigma_{f}\right]\left[\left(R_{v \text { min }}\right)^{s}+\left(R_{v \max }\right)^{s}\right] .
\end{aligned}
$$


Then the damage evolution equation for prediction of notched specimen becomes:

$$
\begin{aligned}
\frac{\delta D}{\delta N} & =\frac{2(1+v) \sigma_{f}^{2 s}}{3 E(1-\beta)(2 E S)^{s}}\left[K_{T} \Delta \sigma-2 \sigma_{f}\right] \times \\
& \times\left[\left(R_{v \text { min }}\right)^{s}+\left(R_{v \text { max }}\right)^{s}\right] .
\end{aligned}
$$

The number of cycles to failure ND under the cycle loading is obtained by integrating Eq. (12).from $D=0$ (initial undamaged state) to $D c$ (macrocrack initiation).

So the total life of notched specimen is:

$$
N_{R}=N_{0}+N_{D}
$$

\section{Calibration of material parameters for LC4 aluminum}

The static properties of LC4 aluminum can be consulted from handbook [20] and the chemical composition and the mechanics properties are presented in Tables 1 and 2 . In the modified damage evolution law, there are four material parameters $\alpha, m, S, s$ need to be demarcated. First, four sets of high cycle fatigue experimental data of standard specimens from handbook [20] are determined. Then the least square method is used [21-22] and the optimum values of these parameters can be obtained which are listed in Table 3.

Table 1

Chemical composition of LC4 aluminum

\begin{tabular}{|c|c|c|c|c|c|c|c|}
\hline $\mathrm{Cu}$ & $\mathrm{Mg}$ & $\mathrm{Zn}$ & $\mathrm{Si}$ & $\mathrm{Fe}$ & $\mathrm{Cr}$ & $\mathrm{Mn}$ & $\mathrm{Al}$ \\
\hline 1.60 & 2.12 & 6.70 & 0.14 & 0.34 & 0.13 & 0.28 & 88.69 \\
\hline
\end{tabular}

Table 2

Static properties of LY7075T75 aluminum alloy material

\begin{tabular}{|c|c|c|c|}
\hline$E, \mathrm{GPa}$ & $v$ & $\sigma b, \mathrm{MPa}$ & $\sigma s, \mathrm{MPa}$ \\
\hline 73 & 0.33 & 549 & 494 \\
\hline
\end{tabular}

Table 3

Material parameters of the modified damage law

\begin{tabular}{|c|c|c|c|}
\hline$\alpha$ & $m$ & $S$ & $s$ \\
\hline 0.15 & 5.5695 & 12.639 & 1.1 \\
\hline
\end{tabular}

\section{The applicability validation of the proposed method}

The geometric profile of the notched specimen for verification of the modified damage law is shown in Fig. 2.

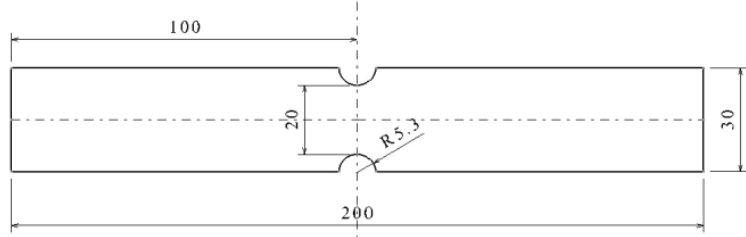

Fig. 2 The geometric profile of notched specimen
The matlab procedure is conducted to predict the fatigue life. The fatigue load, stress ratio and the calculation results under different stress level are shown in Table 4 . The fatigue experimental data of notched specimens are from handbook [20]. From Table 4, we can see that within the scope of wide stress ratio $(-1 \sim 0.68)$, the maximum error between theoretical lives and experimental lives is about $30 \%$.

Table 4

Comparisons between experimental and numerical lives

\begin{tabular}{|c|c|c|c|c|}
\hline $\begin{array}{c}\text { Stress } \\
\text { ratio }\end{array}$ & $\begin{array}{c}\sigma_{\max }, \\
\mathrm{Mpa}\end{array}$ & $\begin{array}{c}\text { Experimental } \\
\text { life }\end{array}$ & $\begin{array}{c}\text { Numerical } \\
\text { results }\end{array}$ & Error, \% \\
\hline-1 & 170 & 17910 & 20633 & 15.2 \\
\hline-1 & 130 & 50040 & 39732 & 20.6 \\
\hline-0.39 & 230 & 16250 & 15374 & 5.4 \\
\hline-0.33 & 210 & 24550 & 20299 & 17.3 \\
\hline-0.07 & 150 & 93130 & 65145 & 30.0 \\
\hline 0 & 280 & 16850 & 17493 & 3.8 \\
\hline 0.12 & 250 & 35000 & 27833 & 20.5 \\
\hline 0.27 & 220 & 68410 & 52788 & 22.8 \\
\hline 0.27 & 330 & 16740 & 16782 & 0.25 \\
\hline 0.31 & 320 & 19360 & 19633 & 1.4 \\
\hline 0.45 & 290 & 50000 & 36572 & 26.8 \\
\hline 0.68 & 250 & 578200 & 462189 & 20.1 \\
\hline
\end{tabular}

On the basis of theoretical model, NN method is adopted to modify and predict the real life of material. The network structure is composed of 3 layers: input layer, hidden layer and output layer, shown in Fig. 3. The input layer is consisted of two dimension data: the stress radio and the maximum stress $\left(R, \sigma_{\max }\right)$. The output data is the relative deviation between the theoretical life $N_{R}$ and the actual life $N_{A}$, expressed as $\bar{N}=\left(N_{A}-N_{R}\right) / N_{R}$.

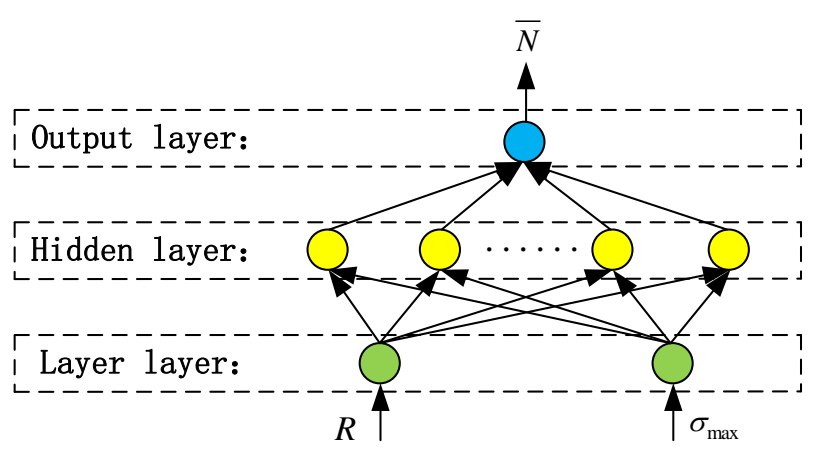

Fig. 3 The structure of the BPNN model

The learning process is divided into two parts: the data collection stage and the BPNN training stage. The training data is obtained through lots of experiments. Under a given radio $R$ and the maximum stress $\sigma_{\max }$, we will calculate the theoretical material life by the Eq. (13), and measure the actual life $N_{A}$ by the experimental equipment. The corresponding relative deviation $\bar{N}$ and the input data will be recorded in the knowledge base. When the experimental quantity is accumulated to a certain degree, the data will be imported into the established neural network for training. The input dimension $i$ is 2 , and the output dimension $o$ is 1 . The neuron numbers $h$ in the hidden layer is estimated by 2 steps: (1) Calculating the value range of $h$ by the empirical formula shown in Eq. (14), in 
which $\alpha$ is set as an integer between 1 to 10. (2) Choosing the value of $h$ corresponding to the local optimum prediction effect by exhaustive method. After a number of attempts and tests, the hidden dimension $h$ is given as 12 .

$$
h=\sqrt{i+o}+\alpha
$$

The training set is randomly divided into 3 parts: $80 \%$ for training, $10 \%$ for test and $10 \%$ for verification. There are no intersection between the training set and the test set. The input data $R$ and $\sigma_{\max }$ will be firstly being standardized into interval $(0,1)$ before imported into the hidden layer. The life prediction task has high requirements both on the performance of interpolation and external expansion ability for the network model, so the effect of standardization is better than normalization for data preprocessing. The learning rate is set as 0.01 , and the training descent curve is shown in Fig. 4. After about 40 epoches of iteration, the error will be reduced and converged to about 0.002 .

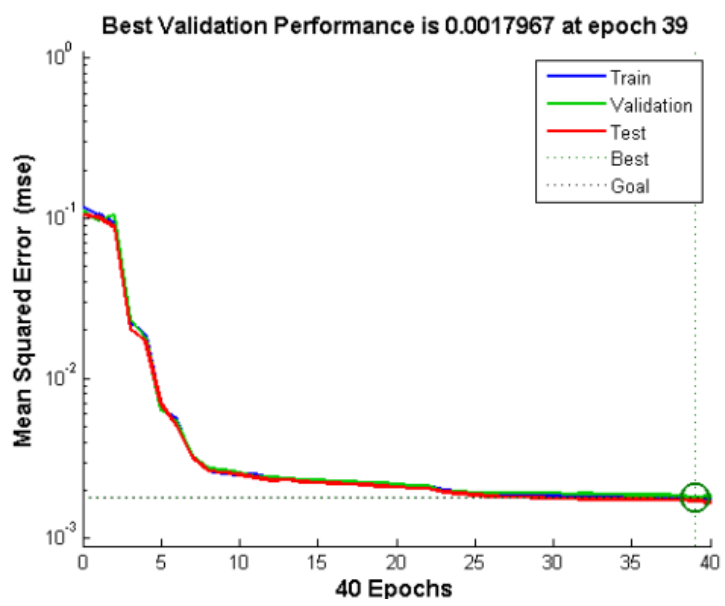

Fig. 4 The descent converged curve during training
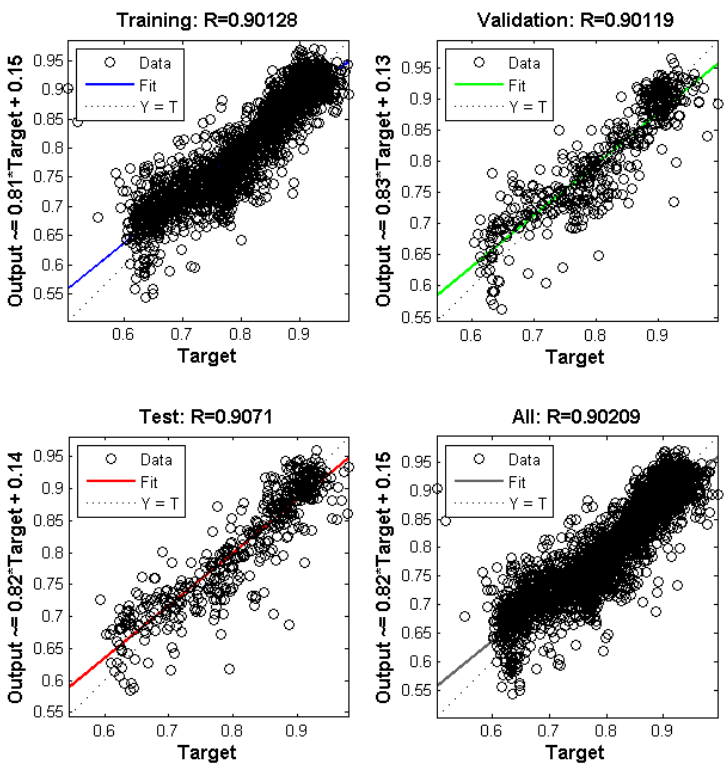

Fig. 5 The regression result for training, test and verification

On the basis of data training, we will analysis the regression relationship between the inputs and output. The regression gradient slope which is more close to 1 means a better fitting effect. The regression coefficient for training, test and verification all exceeds 0.90 and are shown in the Fig. 5. The overall distribution of statistical points is relative disperse, but also can show the trend and the shape of a straight line. Taking into consideration that the actual life is influenced by the complicated factors(such as the material property, the experiment condition, and the flawed theoretical model), the regression result of the deviation NN model is suitable and acceptable.

In a word, the framework of the material life prediction model is given in the Fig. 6. The model consists of two parts: the theoretical model for basic calculation and the NN based deviation model for value modification. Table 1 shows some experiment data for the verification test. The theoretical values $N_{R}$, the modified values by NN model $N_{F}$ and the actual measurement values $N_{A}$ by experiment are recorded in the list. The corresponding relative errors $e_{R}$ and $e_{F}$ are also counted and calculated, and expressed as Eq. (15) shows.

$e_{R}=\frac{N_{R}-N_{A}}{N_{R}} \times 100.0 \%, e_{F}=\frac{N_{F}-N_{A}}{N_{F}} \times 100.0 \%$.

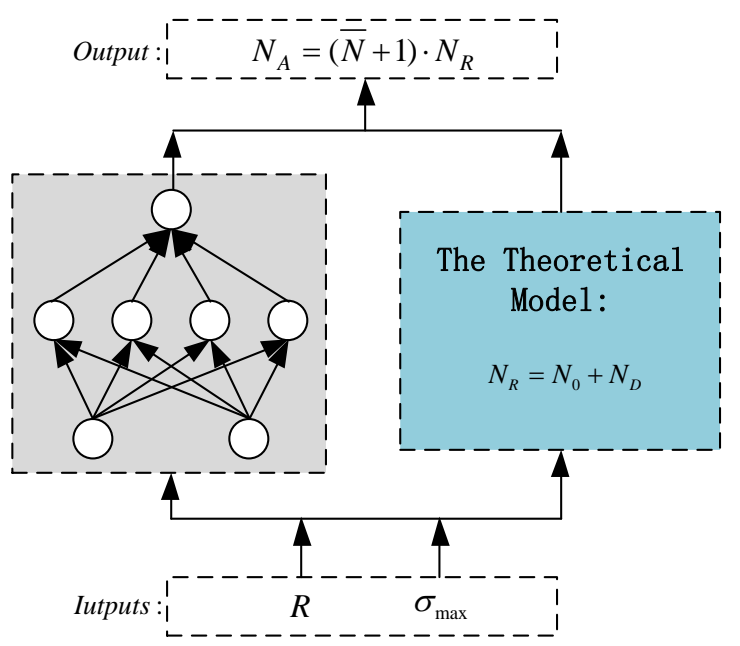

Fig. 6 The material life prediction model combined with the modified damage law and the NN method

Table 5

The comparisons between NF and NR

\begin{tabular}{|c|c|c|c|c|c|c|}
\hline \multicolumn{7}{|c|}{ Verification Test } \\
\hline$R$ & $\sigma_{\max }$ & $N_{A}$ & $N_{R}$ & $e_{R}, \%$ & $N_{F}$ & $e_{F}, \%$ \\
\hline-1 & 170 & 17910 & 20633 & 13.1 & 18910 & 5.2 \\
\hline-1 & 130 & 50040 & 39732 & -25.9 & 45670 & -9.5 \\
\hline-1 & 100 & 86260 & 71628 & -20.4 & 88960 & 3.0 \\
\hline-0.39 & 230 & 16250 & 15374 & -5.6 & 15280 & -6.3 \\
\hline-0.33 & 210 & 24550 & 20299 & -20.9 & 23220 & -5.7 \\
\hline-0.22 & 180 & 46420 & 33086 & -40.3 & 41710 & -11.2 \\
\hline-0.07 & 150 & 93130 & 65145 & -42.9 & 84270 & -10.5 \\
\hline 0 & 280 & 16850 & 17493 & 3.6 & 16840 & -0.1 \\
\hline 0.12 & 250 & 35000 & 27833 & -25.7 & 33110 & -5.7 \\
\hline 0.27 & 220 & 68410 & 52788 & -29.5 & 67300 & -1.6 \\
\hline 0.27 & 330 & 16740 & 16782 & 0.2 & 14150 & -18.3 \\
\hline 0.31 & 320 & 19360 & 19633 & 1.3 & 18440 & -4.9 \\
\hline 0.45 & 290 & 50000 & 36572 & -36.7 & 43350 & -15.3 \\
\hline 0.68 & 250 & 578200 & 462189 & -25.1 & 594170 & 2.6 \\
\hline \multicolumn{3}{|c|}{ The relative error: } & $e_{R}:$ & \pm 20.85 & $e_{A}:$ & \pm 7.12 \\
\hline
\end{tabular}


As indicated in the Table 5, the average relative error for $e_{R}$ is $\pm 20.85 \%$, while the average relative error for $e_{F}$ is $\pm 7.12 \%$. The statistic relationship between the relative errors $\left(e_{R}, e_{A}\right)$ and the inputs $\left(R, \sigma_{\max }\right)$ are shown in Figs. 7, a and b respectively. The red region means a relative high error percent (exceeds $\pm 20 \%$ ), while the blue region means the error percent is less than $\pm 10 \%$. Compared with the simply use of the theoretical model, the results after NN modified will be more close to the actual values in whole, which means that the combination between the modified damage law with the fuzzy neural network method can be effectively applied for fatigue live prediction of notched specimen in high cycle fatigue.

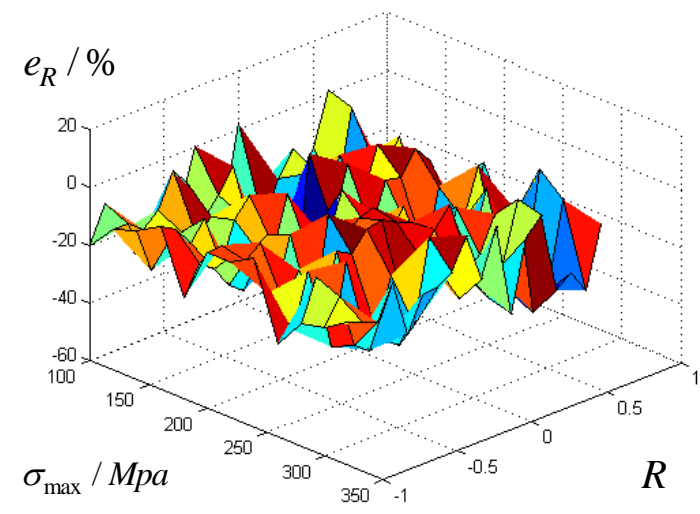

a

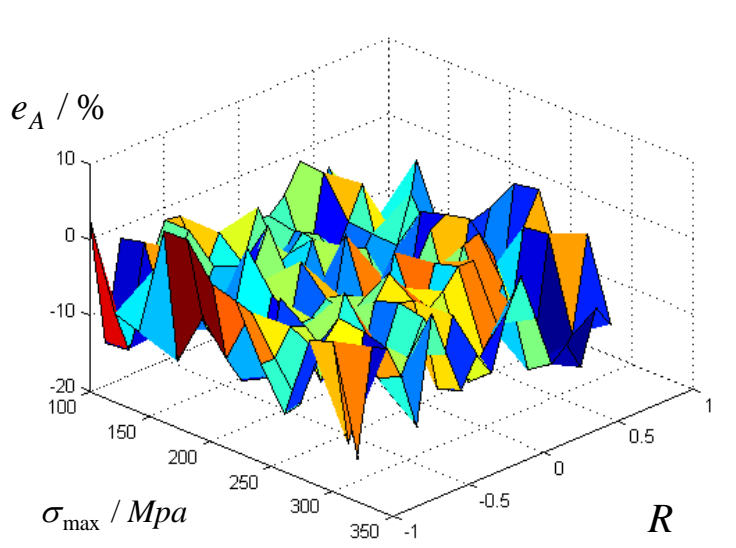

b

Fig. 7 The statistic relationship between the relative errors and the inputs predicting by two methods: a - the relative error statistic simply by theoretical model; $\mathrm{b}$ - he relative error statistic by theoretical model and NN model

Fig. 7, a shows the results simply by the theoretical model; Fig. 7, b shows the results by the combination model of modified damage law and NN method. The red region means a relative high error percent $(> \pm 20 \%)$, while the blue region means the error percent is less than $\pm 10 \%$.

\section{Conclusions remarks}

In this paper, we attempt to combine the damage law with the neural network method to give a more reliable material life in the whole. Some important conclusions are summarized as follows:

(1) A modified damage law for fatigue life pre- diction of notched specimen in high cycle fatigue based on the fatigue damage evolution equation of Lemaitre is proposed. The predicted fatigue lives of notched specimens tally with the fatigue experimental results.

(2) By using the least square method, material parameters of damage evolution equation are demarcated according to the fatigue experimental data of LC4 aluminum, and the practical validation of modified damage law is conducted.

(3) On the basis of (1) and (2), the fuzzy neural network method is adopted to optimize the theoretical model. The trained BPNN model is able to effectively correct the results calculated by the modified damage law. Compared with the predicted results of theoretical model, the combined method of modified damage law and fuzzy neural network can predict the fatigue life with a less average relative error.

\section{References}

1. Zhan, Z.; Hu, W.; Zhang, M.; Zhu, Y.; Meng, Q. 2013. Experimental method for and theoretical research on defect tolerance of fixed plate based on damage mechanics, Chinese Journal of Aeronautics 26(5): 1195-1201. http://dx.doi.org/10.1016/j.cja.2013.04.017.

2. Suresh, S. 1998. Fatigue of materials, Cambridge University Press. http://dx.doi.org/10.1017/CBO9780511806575.

3. Lemaitre, J.; Chaboche, J.L. 1990. Mechanics of solid materials, Cambridge University Press. http://dx.doi.org/10.1061/(ASCE)0733-9399(1993)119: 3(642.2).

4. Zhan, Z.; Hu, W.; Zhang, M.; Meng, Q. 2015. The fatigue life prediction for structure with surface scratch considering cutting residual stress, initial plasticity damage and fatigue damage, International Journal of Fatigue 74: 173-182.

http://dx.doi.org/10.1016/j.ijfatigue.2015.01.011.

5. Chaboche, J.L. 1981. Continuous damage mechanics-a tool to describe phenomena before crack initiation, Nuclear Engineering and Design 64(2): 233-247. http://dx.doi.org/10.1016/0029-5493(81)90007-8.

6. Chaboche, J.; Lesne, P. 1988. A non-linear continuous fatigue damage model, Fatigue $\&$ fracture of engineering materials \& structures 11(1): 1-17. http://dx.doi.org/10.1111/j.1460-2695.1988.tb01216.x.

7. Lemaitre, J.; Plumtree, A. 1979. Application of damage concepts to predict creep-fatigue failures, Journal of Engineering Materials and Technology 101(3): 284-292.

http://dx. doi.org/10.1115/1.3443689.

8. Zhan, Z.; Hu, W.; Zhang, M.; Meng, Q. 2015. A study on the effect of surface defect on the fatigue performance of metal component based on damage mechanics, Mechanics 21(1): 5-10. http://dx.doi.org/10.5755/j01.mech.21.1.7923.

9. Zhan, Z.; Hu, W.; Zhang, M.; Meng, Q. 2015. Revised damage evolution equation for high cycle fatigue life prediction of aluminum alloy LC4 under uniaxial loading, Applied Mathematics and Mechanics 36(9): 1185-1196.

http://dx. doi.org/10.1007/s10483-015-1970-6.

10. Movaghghar, A.; Lvov, G.I. 2012. A method of esti- 
mating wind turbine blade fatigue life and damage using continuum damage mechanics, International Journal of Damage Mechanics 21(6): 810-821. http://dx.doi.org/10.1177/1056789511419693.

11. Zhan, Z.X.; Hu, W.P.; Zhang, M.; Meng, Q. 2012. The Fatigue Life Prediction Problem for Fixed Plate of Helicopter, Advanced Materials Research, Trans Tech Publications 452: 1164-1168. http://dx.doi.org/10.4028/www.scientific.net/AMR.452 $-453.1164$.

12. Gao, J.; He, Q.; Zhu, M.; Liang, H.; Guo, X. 2015. Design of the multiple Neural Network compensator for a billiard robot, International Conference on Networking, Sensing and Control: IEEE. http://dx.doi.org/10.1109/ICNSC.2015.7116003.

13. Gebraeel, N.; Lawley, M.; Liu, R.; Parmeshwaran, V. 2004. Residual life predictions from vibration-based degradation signals: a neural network approach. IEEE Transactions on Industrial Electronics 51(3): 694-700. http://dx.doi.org/10.1109/TIE.2004.824875.

14. Cao, L.; Li, S.; Shangguan, Z. 2008. Damage identification of mechanical system with artificial neural networks, Key Engineering Materials 385-387, 877-880. http://dx.doi.org/10.4028/www.scientific.net/KEM.385 $-387.877$.

15. Mariani, C.; Venini, P.; Nascimbene, R. 2003. Neural networks in computational damage mechanics; Computational Fluid \& Solid Mechanics, 426-428. http://dx.doi.org/10.1016/b978-008044046-0.50105-6.

16. Liao, X.; Xu, W.; Gao, Z. 2008. Application of Artificial Neural Network to Forecast the Tensile Fatigue Life of Carbon Material. Key Engineering Materials 385-387, 533-536.

http://dx.doi.org/10.4028/www.scientific.net/KEM.385 -387.533 .

17. Srivastava, S.; Roy, A.K.; Kumar, K. 2014. Design analysis of Mixed Flow Pump Impeller Blades Using ANSYS and Prediction of its Parameters using Artificial Neural Network, Procedia Engineering 97(4): 2022-2031. http://dx.doi.org/10.1016/j.proeng.2014.12.445.

18. Armaghani, D.J.; Mohamad, E.T.; Momeni, E.; Monjezi, M.; Narayanasamy, M.S. 2015. Prediction of the strength and elasticity modulus of granite through an expert artificial neural network, Arabian Journal of Geosciences 9(1): 1-16. http://dx.doi.org/10.1007/s12517-015-2057-3.

19. Lemaitre, J.; Desmorat, R. 2005. Engineering dam- age mechanics. Berlin Heidelberg: Springer.

20. Wu, X. 1996. Handbook of mechanical properties of aircraft structural metals. China aviation industry press.

21. Zhan, Z.; Hu, W.; Meng, Q.; Shi, S. 2015. Continuum damage mechanics-based approach to the fatigue life prediction for 7050-T7451 aluminum alloy with impact pit, International Journal of Damage Mechanics 25(7): $943-966$ http://dx.doi.org/10.1177/1056789515608232.

22.Zhan, Z.; Hu, W.; Meng, Q.; Wang, K. 2015. A damage mechanics-based fatigue life prediction approach for 30crmnsia alloy steel with impact defect. Reliability Systems Engineering (ICRSE): IEEE 1-5. http://dx.doi.org/10.1109/ICRSE.2015.7366484.

Jiaying Gao, Peng Li, Qiuyang He

\section{A MODIFIED DAMAGE LAW WITH THE FUZZY \\ NEURAL NETWORK METHOD FOR CRACK INITIATION LIFE PREDICTION OF NOTCHED SPECIMEN}

S u m m a r y

It is difficult to predict the fatigue life of notched specimen due to the complexity of notch effect. Based on the continuum damage mechanics theory of Lemaitre,a modified damage law for fatigue life prediction of notched specimen combined with the fuzzy neural network method is proposed to obtain a more reliable life. According to the fatigue experimental data, the material parameters in the damage evolution equation are demarcated. Then the fatigue life prediction of notched specimen is conducted. At last, after the stage pf experiment data collection and NN training, the established NN model will be able to reduce the relative error from $20.85 \%$ to about $\% 7.12$ in the verification test. Although the life prediction after being modified may not be accurate in every local region, the combination between the theoretical method and the fuzzy neural network method will obtain a more reliable material life in the whole.

Keywords: Modified damage law, Fuzzy neural network, Crack initiation, Notched specimen, experimental verification.

Received May 06, 2016

Accepted December 07, 2017 